\title{
Comparison of the effects of millimeter wave irradiation, general bath heating, and localized heating on neuronal activity in the leech ganglion
}

\author{
Sergii Romanenko $^{\text {ab }}$, Peter H. Siegel ${ }^{\mathrm{a}}$, Daniel A. Wagenaar ${ }^{\mathrm{a}}$, Victor Pikov ${ }^{\mathrm{b}^{*}}$ \\ ${ }^{a}$ California Institute of Technology, Pasadena, CA 91125 USA \\ ${ }^{\mathrm{b}}$ Huntington Medical Research Institutes, Pasadena, CA 91105 USA
}

\begin{abstract}
The use of electrically-induced neuromodulation has grown in importance in the treatment of multiple neurological disorders such as Parkinson's disease, dystonia, epilepsy, chronic pain, cluster headaches and others. While electrical current can be applied locally, it requires placing stimulation electrodes in direct contact with the neural tissue. Our goal is to develop a method for localized application of electromagnetic energy to the brain without direct tissue contact. Toward this goal, we are experimenting with the wireless transmission of millimeter wave (MMW) energy in the 10-100 $\mathrm{GHz}$ frequency range, where penetration and focusing can be traded off to provide non-contact irradiation of the cerebral cortex. Initial experiments have been conducted on freshly-isolated leech ganglia to evaluate the real-time changes in the activity of individual neurons upon exposure to the MMW radiation. The initial results indicate that low-intensity MMWs can partially suppress the neuronal activity. This is in contrast to general bath heating, which had an excitatory effect on the neuronal activity. Further studies are underway to determine the changes in the state of the membrane channels that might be responsible for the observed neuromodulatory effects.
\end{abstract}

Keywords: Neuron, action potential, leech, millimeter waves, non-invasive, brain, GHz, terahertz.

\section{INTRODUCTION}

Modulation of neuronal activity and excitability has been utilized for treating Parkinson's disease, dystonia, epilepsy, chronic pain, cluster headache, and other neurological disorders [1]. Amongst the available neuromodulation approaches, electrical stimulation is presently the most common technique for inducing neuronal excitatory and inhibitory effects used in the treatment of these disorders [2]. Despite clinical availability of electrical stimulation devices, their adoption by neurosurgeons and patients has lagged, in part because of the considerable risks associated with the surgical procedures for their insertion, and the potential for tissue damage during long-term implantation [3].

In an effort to reduce the risks and inherent invasiveness of surgically implanted electrodes, we are exploring the potential application of non-contact focused electromagnetic energy on the nervous tissue. In an earlier study using rat-pup cortical slices, we demonstrated the feasibility of directly affecting neuronal activity using this approach [4]. To directly study the mechanisms of the observed neuromodulatory effects of the MMWs on neurons, we have begun working with freshly-dissected leech segmental ganglia, as they provide a simple biological preparation for the neurophysiological evaluation of specific identifiable neurons over an extended experimentation period. Previously, it has been reported [5] that focused microwaves $(1-5 \mathrm{GHz})$ can inhibit the firing rate of individual ganglionic neurons in another invertebrate, Aplysia. In contrast, an equivalent amount of observed temperature rise by general bath heating produced an increase in the firing rate in a variety of poikilothermic animals, such as Aplysia [5], cockroach [6], and fly [7], as well as in homeothermic animals, such as the cat [8]. The heating-induced increase in the firing rate has been postulated to be due to faster opening dynamics of the voltage-gated $\mathrm{K}^{+}$and $\mathrm{Na}^{+}$channels in the cell membrane [9], as well as changes in the membrane capacitance [10]. Recently, the ability of localized infrared heating to induce neuronal

Terahertz and Ultrashort Electromagnetic Pulses for Biomedical Applications, edited by Gerald J. Wilmink, Bennett L. Ibey, Proc. of SPIE Vol. 8585, 85850N · (c) 2013 SPIE · CCC code: 1605-742/13/\$18 · doi: 10.1117/12.2006504 
activation was utilized for the stimulation of auditory, vestibular, and peripheral nerves [11]. Considering these reported excitatory effects of both general and localized heating, which contrast with the inhibitory effects observed with the application of MMWs, we have performed a direct comparison of the effects of 60-GHz MMW irradiation with heating from both a rise in bath temperature and the application of localized red light on individual neurons in the leech segmental ganglion.

\section{METHODS}

Adult medicinal leeches Hirudo verbana were obtained from Niagara Medical Leeches, Inc. (Westbury, NY, USA). Groups of animals (20-30) were kept in glass aquaria with artificial pond water (36 mg/L Instant Ocean salts, Aquarium Systems, Mentor $\mathrm{OH}$ ), in a temperature-controlled room at $16^{\circ} \mathrm{C}$ and a $12: 12$ hour light:dark cycle. The leach was anesthetized in ice-cold leech saline and pinned down in a Sylgard-coated dissection chamber. Leech saline had the following composition (in $\mathrm{mM}$ per liter): $\mathrm{NaCl}-115, \mathrm{KCl}-4, \mathrm{CaCl}_{2}-1.8, \mathrm{MgCl}_{2}-2$, HEPES - 10, Glucose - 10 (all chemicals from Sigma-Aldrich, St Louis, MO, USA). For the ganglion dissection, a medial dorsal longitudinal incision was made, and the blood was flushed away. Connective tissue and the blood vessel overlaying the ganglion chain were incised, and the individual ganglia were dissected from the mid-body segments M6-M12. Dissected ganglia were transferred to a Petri dish, pinned down in paraffin with six stainless steel pins (dorsal side up) to secure the connectives and lateral roots, and kept in the refrigerator $\left(+4^{\circ} \mathrm{C}\right)$ for several hours until used in the experiment. The Petri dish was placed under an upright microscope (BX-FM, Olympus) and filled with leech saline so that the ganglion was covered by a 1-mm-thick fluid layer. The total amount of saline was approximately $5 \mathrm{ml}$. The ganglion was illuminated with a white LED light though pseudo-DIC microscope optics; and a 10x objective was employed for distinguishing the cell types. Intracellular recordings were performed mostly from two types of neurons: the Retzius and the AP cells [12]. These neuronal types are large and easily identifiable even when viewed through the outer capsule, making them a convenient choice for penetration with the intracellular electrode.

For intracellular recording, sharp electrodes were fabricated by pulling borosilicate capillary glass $(0.75 \mathrm{~mm}$ inner diameter, $1 \mathrm{~mm}$ outer diameter, cat. \# 615000, A-M Systems, Sequim, WA, USA) using a micropipette puller (P-97, Sutter Instrument Company, Novato, CA, USA). The electrodes were filled with $3 \mathrm{M} \mathrm{K}$-Acetate and $20 \mathrm{mM} \mathrm{KCl}$ unbuffered solution, and their initial resistance was in the range of 22-27 M 2 . All electrophysiological recordings were performed using a microelectrode amplifier (Axoclamp 900A, Molecular Devices, Sunnyvale, CA, USA) in the currentclamp mode with a holding current of $0 \mathrm{nA}$, then digitized at $20 \mathrm{kHz}$ using a data acquisition system (DigiData 1440A, Molecular Devices), and software (Clampex 10, Molecular Devices). Electrophysiological recordings were performed using the gap-free mode and episodic stimulation, and then processed with the data analysis software (Clampfit 10, Molecular Devices). The action potential shape was evaluated using the following parameters: resting membrane potential (RMP) and half-width (the action potential span from the pre-peak to post-peak half-amplitude). The leech saline was modified by adding $0.1 \mathrm{mM}$ reserpine (Sigma-Aldrich) to reduce the synaptic coupling among the neurons in the ganglion [13]. The addition of reserpine did not alter the rhythmic spontaneous firing of the probed neurons, and the action potential parameters remained within the physiological range: the RMP of the Retzius cells sat at $-43.0 \pm 1.1 \mathrm{mV}$, the action potential amplitude was $35.1 \pm 2.6 \mathrm{mV}$, the half-width was $4.2 \pm 0.25 \mathrm{msec}$, and the firing rate was $1.0 \pm 0.15$ $\mathrm{Hz}$.

The MMW exposure system (Figs. 1-3) consisted of a synthesized microwave source for 17-23 GHz (HP 83650L, Agilent Technologies, Santa Clara, CA), a 4x frequency-multiplying stage (HP 83557A, Agilent Technologies) or an active quadrupler (AMC15, Millitech Corp., Deerfield, MA), followed by a $20 \mathrm{~dB}$ amplifier at $60 \mathrm{GHz}$ (AMP-15, Millitech, Northampton, MA, USA) to generate continuous wave power between 0 and $100 \mathrm{~mW}$. Power levels could be controlled using internal or external attenuators, and was continuously monitored with both a $60 \mathrm{GHz}$ waveguide mixer (47344H-1200, Hughes Electronics (now Raytheon), El Segundo, CA, USA) and a calibrated absolute thermoelectric power meter (ML83A, Anritsu, Kanagawa, Japan). Coupling to the ganglion was accomplished through a single-mode open-ended rectangular waveguide (WR15 designation for single-mode operation in the $50-75 \mathrm{GHz}$ band, $3.8 \times 1.9 \mathrm{~mm}$ aperture) placed $1 \mathrm{~mm}$ below the Petri dish. The path of the MMW radiation after exiting the waveguide was through a central opening in the microscope stage, the polystyrene Petri dish bottom (1 mm thick), the low-RF-loss paraffin holding the ganglion pins ( $3.2 \mathrm{~mm}$ thick), and then directly into the pinned-out ganglion ( $0.2 \mathrm{~mm}$ thick). The Petri dish was filled with leech saline to a level $1 \mathrm{~mm}$ above the top of the ganglion. The waveguide was aligned with the microscope optical axis so that the peak output power from the guide (MMW beam much broader than the cell cluster) fell across the entire ganglion. The amount of MMW power that actually reached the neuron being probed at the top of 
the ganglion could not be directly measured, as no well-matched or calibrated MMW detector is available at a submillimeter scale or for fluid-immersed measurements. The average power density at the ganglion was instead calculated using a well-established commercial Finite Difference Time Domain (FDTD) simulator (Quickwave, QWED, Warsaw, Poland). Using the setup dimensions, free space wavelength $(5 \mathrm{~mm})$, and complex dielectric permittivity (measured with an Agilent 85070E dielectric probe) of the polystyrene Petri dish $\left(\varepsilon_{\mathrm{r}}=2.53, \sigma=0.008\right)$, paraffin $\left(\varepsilon_{\mathrm{r}}=1.63, \sigma=0\right)$, and leech saline solution $\left(\varepsilon_{\mathrm{r}}=12.4, \sigma=71.26\right)$, the simulator was run with a series of different mesh sizes, frequencies, geometric areas, and other parameter variations, to establish sensitivities and confirm the absence of strong frequencylocalized resonances or variations across the exposed sample area. The ganglion was too small to measure its permittivity directly, so it was estimated to be $\varepsilon_{\mathrm{r}}=12.0, \sigma=60$ based on its high water content and reported data on cortical neurons. As a consequence of the uncertainties in the physical parameters (permittivity and dimensions), the FDTD-estimated power density at various points in the sample has significant error bars ( $\pm 6 \mathrm{~dB})$. The MMW power loss was calculated with the FDTD software, accounting for beam expansion, reflection and refraction at each interface, and absorption in each material from the waveguide output port through the air gap, polystyrene, wax, ganglion and leech saline. The simulated power loss referenced to the rectangular waveguide output was $12.5 \mathrm{~dB}(18 \mathrm{X})$ at the bottom of the ganglion, and $17.8 \mathrm{~dB}(60 \mathrm{X})$ at the top of the ganglion. At the applied power range from 4 to $64 \mathrm{~mW}$, the peak power density at the waveguide port (which is a maximum at the center and rolls off towards the edges of the broad wall) ranges from 53 to $840 \mathrm{~mW} / \mathrm{cm}^{2}$, translating to a range from 0.9 to $14 \mathrm{~mW} / \mathrm{cm}^{2}$ at the top of the ganglion. The duration of MMW irradiation was always 1 minute, and a between-exposure interval of at least 5 minutes was used to avoid the possible effects of cumulative exposure. It should be noted that the IEEE safe exposure limit, adopted by the US, is 1 $\mathrm{mW} / \mathrm{cm}^{2}$ for 6 minutes [14].

Monitoring of the bath temperature was done using a thermistor (TA-29, Thermometrics, Edison, NJ, USA) with a precision of $0.01^{\circ} \mathrm{C}$. During the MMW exposure and general bath heating experiments, the thermistor was placed in the leech saline at $5 \mathrm{~mm}$ distance from the ganglion and at least $3 \mathrm{~mm}$ distance from the bottom of the dish. This assured that all power was fully absorbed in the fluid before interacting with the probe to avoid direct coupling of the MMW energy with the thermistor, an effect that has been reported in the literature [15]. During localized heating, the thermistor was placed against the lateral ganglion surface. For general bath heating, the bath solution was passed through an inline heater (SH-27B, Warner instruments, Hamden, CT, USA), connected to a single-channel heater controller (TC-324B, Warner instruments) with an accuracy of $0.5^{\circ} \mathrm{C}$.

Localized ganglion heating was produced by placing the ganglion in the middle of the microscopic field of view and applying red light at a wavelength of $671 \mathrm{~nm}$ and a power of $0.74 \mathrm{~mW}$ from a DPSS laser (VA-I-N-671, Beijing Viasho Technology, Beijing, China), then focusing the laser beam through the 10x microscope objective to achieve a beam diameter of approximately $120 \mu \mathrm{m}$. The beam passed through $1 \mathrm{~mm}$ of bath solution before reaching the ganglion.

The data are reported as the mean \pm standard deviation.

\section{RESULTS}

First, we evaluated the effects of general bath heating and localized ganglion heating on the neuronal activity at a holding current of $0 \mathrm{nA}$ (Fig. 4). General bath heating was introduced at a rate of $\sim 0.1^{\circ} \mathrm{C} / \mathrm{sec}$, as measured by a thermistor located $5 \mathrm{~mm}$ away from the ganglion. For the localized ganglion heating using the $671 \mathrm{~nm}$ laser, the thermistor was placed adjacent to the ganglion and the measured temperature rise at the ganglion surface reached $0.5^{\circ} \mathrm{C}$ after a 60 -sec-long exposure.

Both the general bath heating and localized red light heating produced significant changes in the neuronal activity, however these changes had opposite polarity (Figs. 4A \& B). After a bath temperature rise of $2.5^{\circ} \mathrm{C}$, the RMP depolarization reached $2.3 \pm 0.36 \mathrm{mV}$ (Fig. 4C), the action potential half-width narrowed by $0.19 \pm 0.08 \mathrm{msec}$ (Fig. 4E), and the firing rate increased by $0.36 \pm 0.24 \mathrm{~Hz}$ (Fig. 4G). The 60-sec-long localized red-light heating produced considerable half-width narrowing by $1.5 \mathrm{msec}$ (Fig. 4F), and opposite effects on RMP and firing rate: RMP hyperpolarization of $2.1 \mathrm{mV}$ (Fig. 4D) and the firing rate decrease of $0.2 \mathrm{~Hz}$ (Fig. 4H). 
Next, we evaluated the effect of $60 \mathrm{GHz}$ MMW exposure on the ganglionic neurons. Several power levels ranging from 4 to $64 \mathrm{~mW}$ at the output waveguide port (translating to power densities from 0.9 to $14 \mathrm{~mW} / \mathrm{cm}^{2}$ at the top of the ganglion) were applied. Fig. 5 shows representative changes in the activity of Retzius cells during 60-sec exposures at two MMW power levels: 4 and $16 \mathrm{~mW}$ (approximately 0.9 and $3.5 \mathrm{~mW} / \mathrm{cm}^{2}$ ). The MMW application produced RMP hyperpolarization (Fig. 5C \& D), slight half-width narrowing (Fig. 5E \& F), and a substantial reduction in the firing rate (Fig. 5G \& H).

The MMW exposures were evaluated in 42 neurons. The average recorded changes for the $16 \mathrm{~mW}\left(3.5 \mathrm{~mW} / \mathrm{cm}^{2}\right)$ exposures were: RMP hyperpolarization of $1.64 \pm 0.21 \mathrm{mV}(1-2 \%)$, half-width narrowing of $0.37 \pm 0.05 \mathrm{msec}(4-9 \%)$, and a firing rate decrease of $0.3 \pm 0.05 \mathrm{~Hz}(20-30 \%)$.

In summary, the RMP and firing rate decreased during localized red-light illumination and MMW exposure, but not during general bath heating; and all heating modalities produced some half-width narrowing.

A question remained as to the amount of heating caused by the MMW radiation. Since it was not possible to directly measure the amount of MMW exposure or its specific absorption rate (SAR) at the position of the probed neuron, we performed a separate thermal calibration using the resistance change with temperature of an electrolyte filled probe. In order to estimate the actual amount of ganglionic heating produced by the MMW exposure, we compared the voltage drop inside the glass electrode during general bath heating and during MMW exposure, when the electrode was positioned just outside the ganglion. Based on the well-understood mechanism of ionic conductivity change with temperature [16], and using the voltage drop relative to the baseline junction potential as a proxy for conductivity, the voltage drop was correlated with the bath temperature during general bath heating (Fig. 6A). Note that the coefficients of the second-degree polynomial fit are rather different from those reported for dilute electrolytes [17], which is not too surprising considering that a highly-saturated (3 M KAc) solution was used.

We then evaluated the voltage drop during MMW exposure at a range of power levels (from 4 to $64 \mathrm{~mW}$ at the waveguide port) and at a range of applied electrode currents (from 0 to $10 \mathrm{nA}$ ) (Fig. 6B). We observed a consistent parasitic effect on the voltage change from the applied current, likely due to increased ionic flow through the pipette. In order to eliminate this confounding effect on the voltage recordings, all experiments with the MMW exposures were performed at a holding current of $0 \mathrm{nA}$.

The empirically established relationship between temperature and voltage drop obtained from the general bath heating experiment (Fig 6A) and the 0-nA data from the MMW experiment (Fig 6B), were used to calibrate the temperature rise during MMW exposure of the ganglion. The results are shown in Fig. 6 C-D for a 15-second MMW exposure at three power levels $(16,32$, and $64 \mathrm{~mW}$ at the waveguide port). As evident from the plots in Fig. 6D, even at a high power level of $32 \mathrm{~mW}\left(7 \mathrm{~mW} / \mathrm{cm}^{2}\right.$ at the top of the ganglion), the MMW-induced heating did not exceed $1{ }^{\circ} \mathrm{C}$. Based on the derived temperature/voltage-drop relationship, we estimate that during the MMW exposure of the leech ganglion, the ganglion heating rate was $0.04{ }^{\circ} \mathrm{C} / \mathrm{mW}$. At such a heating rate, the 60 -sec-long $\mathrm{MMW}$ exposure at $16 \mathrm{~mW}$ causes a ganglion temperature increase of only $0.6{ }^{\circ} \mathrm{C}$. It should be noted that this approach of estimating the ganglion heating slightly overestimates the actual temperature rise as the system reaches a thermal diffusion limit in a fairly short time $(<30$ seconds) as can be observed Fig 6D.

\section{DISCUSSION}

We used ex vivo leech ganglion preparations to evaluate the effects of general bath heating, localized red light ganglionic heating and general MMW exposure on the activity of large individual neurons, namely the Retzius and AP cells. The half-width of action potentials was consistently decreased by all three sources of applied heat, while the RMP and firing rate were oppositely impacted by general bath heating in contrast to both red light and MMW heating. General bath heating produced membrane depolarization and increased firing rate, which are consistent with predictions (i.e. the Goldman-Hodgkin-Katz equation) and previous experimental studies [5-8]. The onset of hyperpolarization and a slowdown of the firing rate by localized red-light $(671 \mathrm{~nm})$ illumination is quite dissimilar from effects of localized infrared $(1870-2120 \mathrm{~nm})$ illumination reported by other groups [10,11, 18]. We hypothesize that the observed differences in the effects of 671-nm and infrared illumination may be due either to differential absorption of the two very different 
wavelengths by the ganglion, to a difference in the produced thermal gradient, or to the time scales used in the different experiments - our experiments use continuous wave exposures rather than pulsed light.

The magnitude of the effects of MMW exposure on RMP and firing rate were similar to those induced by localized 671 $\mathrm{nm}$ light when the optical power was $0.74 \mathrm{~mW}$ and the MMW power was $16 \mathrm{~mW}\left(3.5 \mathrm{~mW} / \mathrm{cm}^{2}\right.$ at the top of the ganglion). The MMW exposure had an opposite effect on RMP and firing rate compared to general bath heating. We speculate that the observed hyperpolarization and firing rate effects at $671 \mathrm{~nm}$ and at $60 \mathrm{GHz}$ may be due to a sharper temporal and/or spatial temperature gradient compared to general bath heating.

Overall, this study demonstrates that low-power MMW exposures, below the recommended safety limits, can modulate the activity of neurons in the leech ganglion and, in contrast to general bath heating, produce an inhibitory, rather than excitatory effect. MMW irradiation offers unique advantages over other methods of neuromodulation as it could be applied through a focused beam onto cortical or spinal tissue with less scattering than optical energy and with perhaps more localized absorption due to the high loss tangent of neuronal tissue. If irradiated through a focusing lens or an externally placed waveguide, MMWs may find an application for modulating neuronal activity in the spinal cord or cerebral cortex without the need for permanently implanted electrodes. 


\section{REFERENCES}

[1] Arle, J. E., and Shils, J. L., [Essential Neuromodulation] Academic Press, 1-491 (2011).

[2] Benabid, A.-L., Wallace, B., Mitrofanis, J. et al., "Therapeutic electrical stimulation of the central nervous system," Comptes Rendus Biologies, 328(2), 177-186 (2005).

[3] Weaver, F. M., Follett, K., Stern, M. et al., "Bilateral deep brain stimulation vs best medical therapy for patients with advanced parkinson disease: A randomized controlled trial," JAMA, 301(1), 63-73 (2009).

[4] Pikov, V., Arakaki, X., Harrington, M. et al., "Modulation of neuronal activity and plasma membrane properties with low-power millimeter waves in organotypic cortical slices," J Neural Eng, 7, 045003 (2010).

[5] Wachtel, H., Seaman, R., and Joines, W., "Effects of low-intensity microwaves on isolated neurons," Ann NY Acad Sci, 247(1), 46-62 (1975).

[6] Janiszewski, J., "The effect of temperature changes on the spontaneous activity in the neural ganglia of the cockroach, Periplaneta americana," J Thermal Biology, 11(4), 191-197 (1986).

[7] Warzecha, A., Horstmann, W., and Egelhaaf, M., "Temperature-dependence of neuronal performance in the motion pathway of the blowfly calliphora erythrocephala," J Exp Biology, 202(22), 3161-3170 (1999).

[8] Klee, M. R., Pierau, F. K., and Faber, D. S., "Temperature effects on resting potential and spike parameters of cat motoneurons," Exp Brain Res, 19(5), 478-492 (1974).

[9] Lee, J. C. F., Callaway, J. C., and Foehring, R. C., "Effects of Temperature on Calcium Transients and Ca2+Dependent Afterhyperpolarizations in Neocortical Pyramidal Neurons," J Neurophysiol, 93(4), 2012-2020 (2005).

[10] Shapiro, M. G., Homma, K., Villarreal, S. et al., "Infrared light excites cells by changing their electrical capacitance," Nat Commun, 3, 736 (2012).

[11] Wells, J. D., Cayce, J. M., Mahadevan-Jansen, A. et al., [Infrared Nerve Stimulation: A Novel Therapeutic Laser Modality] Springer Netherlands, 24 (2011).

[12] Muller, K. J., Nicholls, J. G., and Stent, G. S., [Neurobiology of the leech] Cold Spring Harbor Laboratory, N.Y.(1981).

[13] O'Gara, B., Chae, H., Latham, L. et al., "Modification of leech behavior patterns by reserpine-induced amine depletion," J Neurosci, 11(1), 96-110 (1991).

[14] IEEE, [IEEE Standard for Safety Levels With Respect to Human Exposure to Radio Frequency Electromagnetic Fields, $3 \mathrm{kHz}$ to $300 \mathrm{GHz}$ ] IEEE, Piscataway, NJ(2005).

[15] Alekseev, S. I., and Ziskin, M. C., "Distortion of millimeter-wave absorption in biological media due to presence of thermocouples and other objects," IEEE Trans Biomed Eng, 48(9), 1013-9 (2001).

[16] Kuyucak, S., and Chung, S.-H., "Temperature dependence of conductivity in electrolyte solutions and ionic channels of biological membranes," Biophys Chem, 52(1), 15-24 (1994).

[17] Potter, E. C., [Electrochemistry] The macmillan Company, New York, $26-27$ (1956).

[18] Fried, N., Lagoda, G., Scott, N. et al., "Noncontact stimulation of the cavernous nerves in the rat prostate using a tunable-wavelength thulium fiber laser," J Endourol, 22(3), 409-413 (2008). 


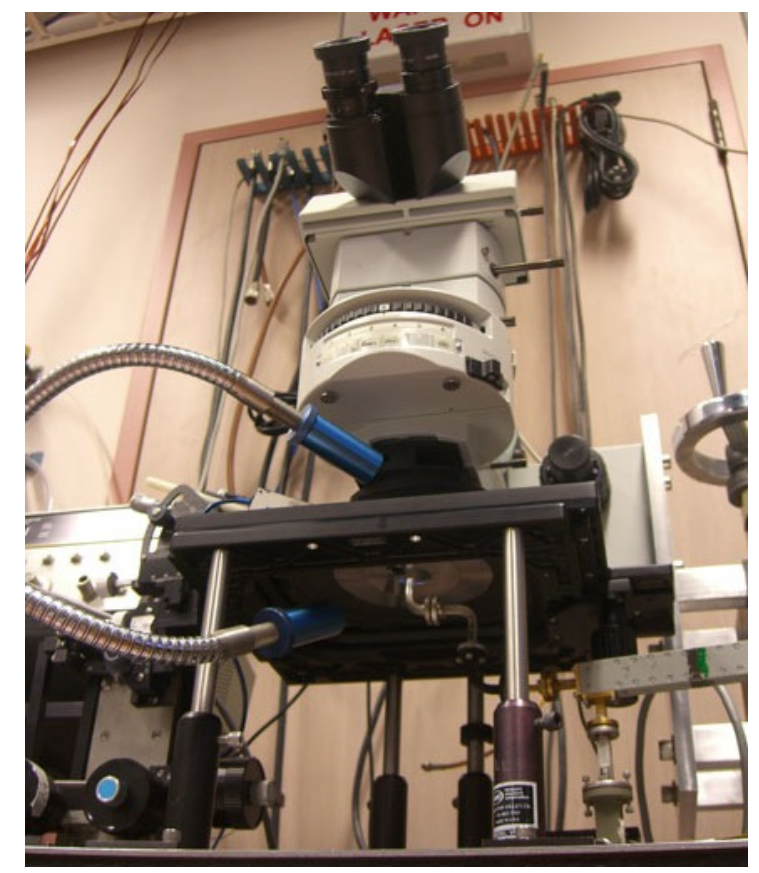

Figure 1. MMW waveguide placed below the recording chamber.

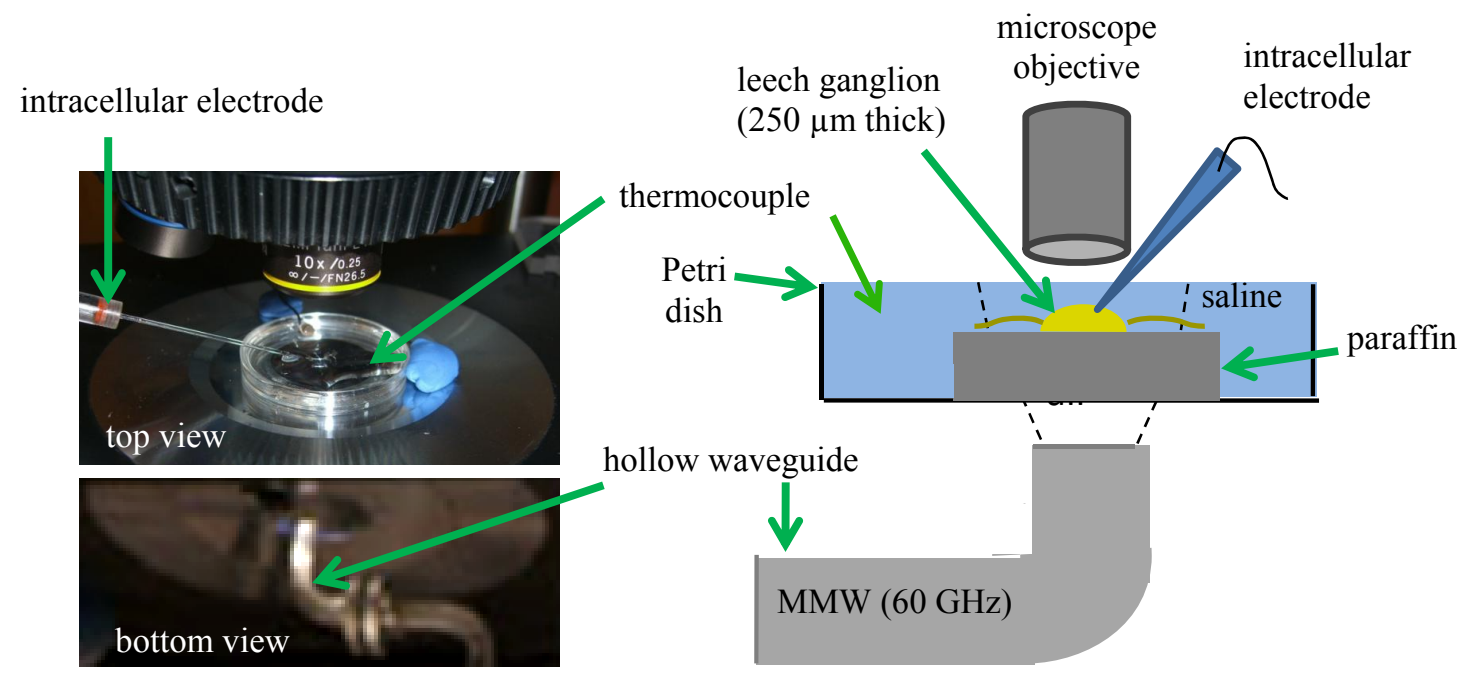

Figure 3. Photo (left) and diagram (right) of experimental set up for MMW illumination of the ganglion 

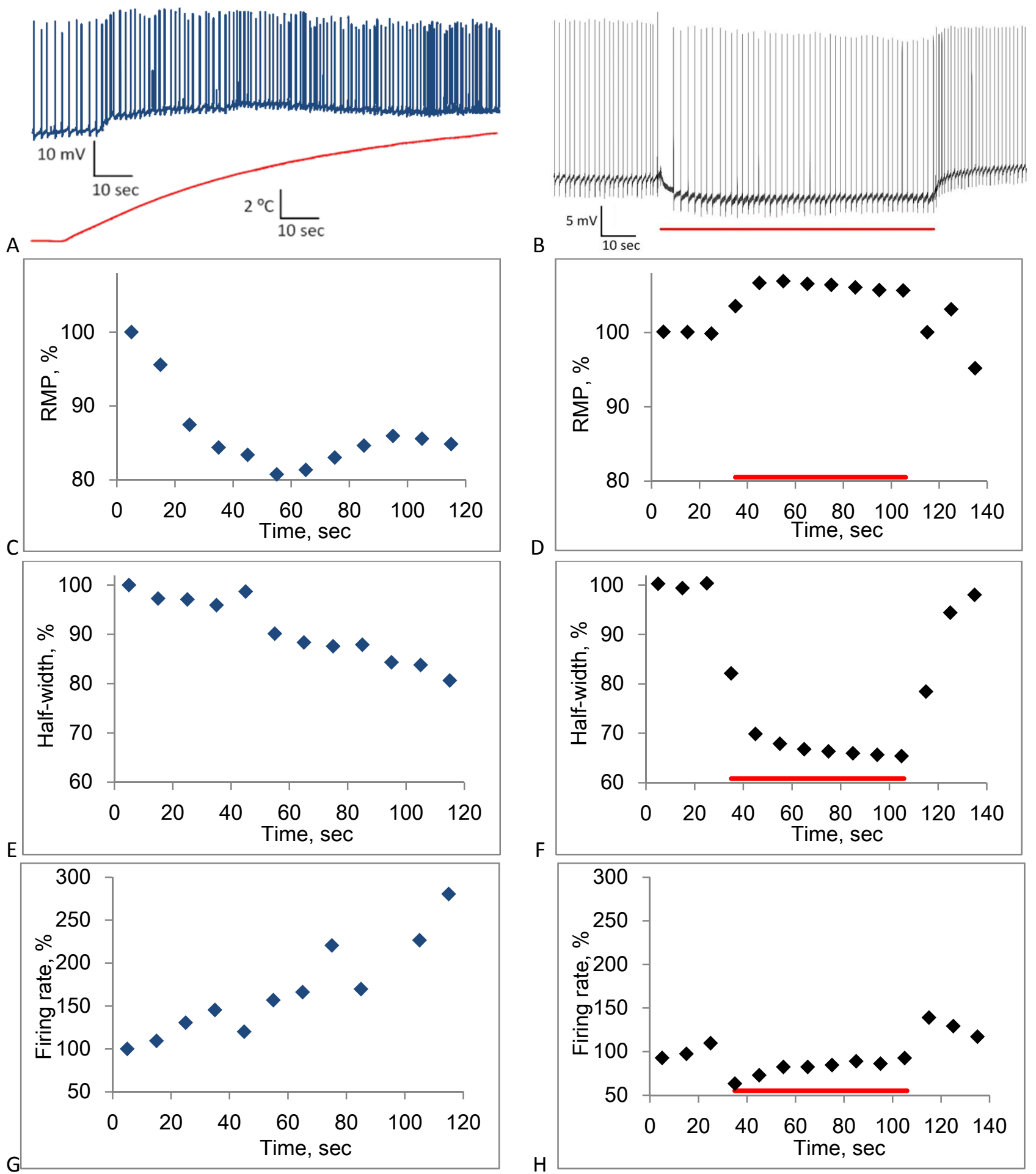
Figure 4. Effects of general bath heating (A, C, E, G) and localized red light illumination $(\mathrm{B}, \mathrm{D}, \mathrm{F}, \mathrm{H})$ on the activity of Retzius
neurons. (A-B): Representative recordings of electrophysiological activity overlaid with the temperature readings. (C-H) Quantification of the key action potential parameters for the recordings, shown in A-B: (C-D) the resting membrane potential, (E-F) half-width, and (G-H) firing rate. The horizontal red bar indicates the duration of bath heating. 

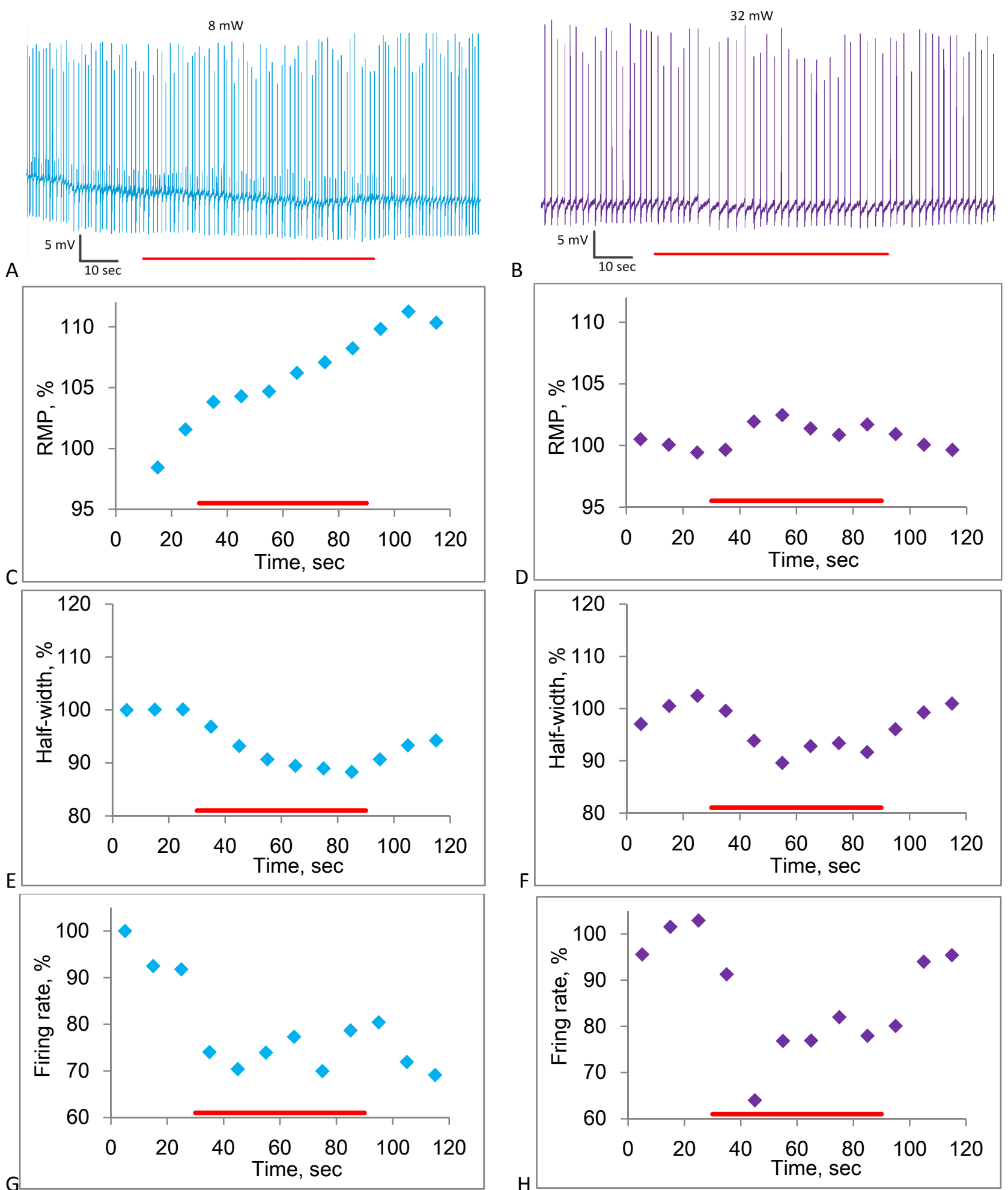

Figure 5. Effects of $60 \mathrm{GHz}$ MMW irradiation at the waveguide port power levels of $4 \mathrm{~mW}(\mathrm{~A}, \mathrm{C}, \mathrm{E}, \mathrm{G})$ and $16 \mathrm{~mW}(\mathrm{~B}, \mathrm{D}, \mathrm{F}, \mathrm{H})$ on the activity of Retzius neurons. (A-B): Representative recordings of electrophysiological activity overlaid with the temperature readings. $(\mathrm{C}-\mathrm{H})$ Quantification of the key action potential parameters for the recordings, shown in A-B: (C-D) the resting membrane potential, (E-F) half-width, and (G-H) firing rate. The horizontal red bar indicates the duration of MMW irradiation. 

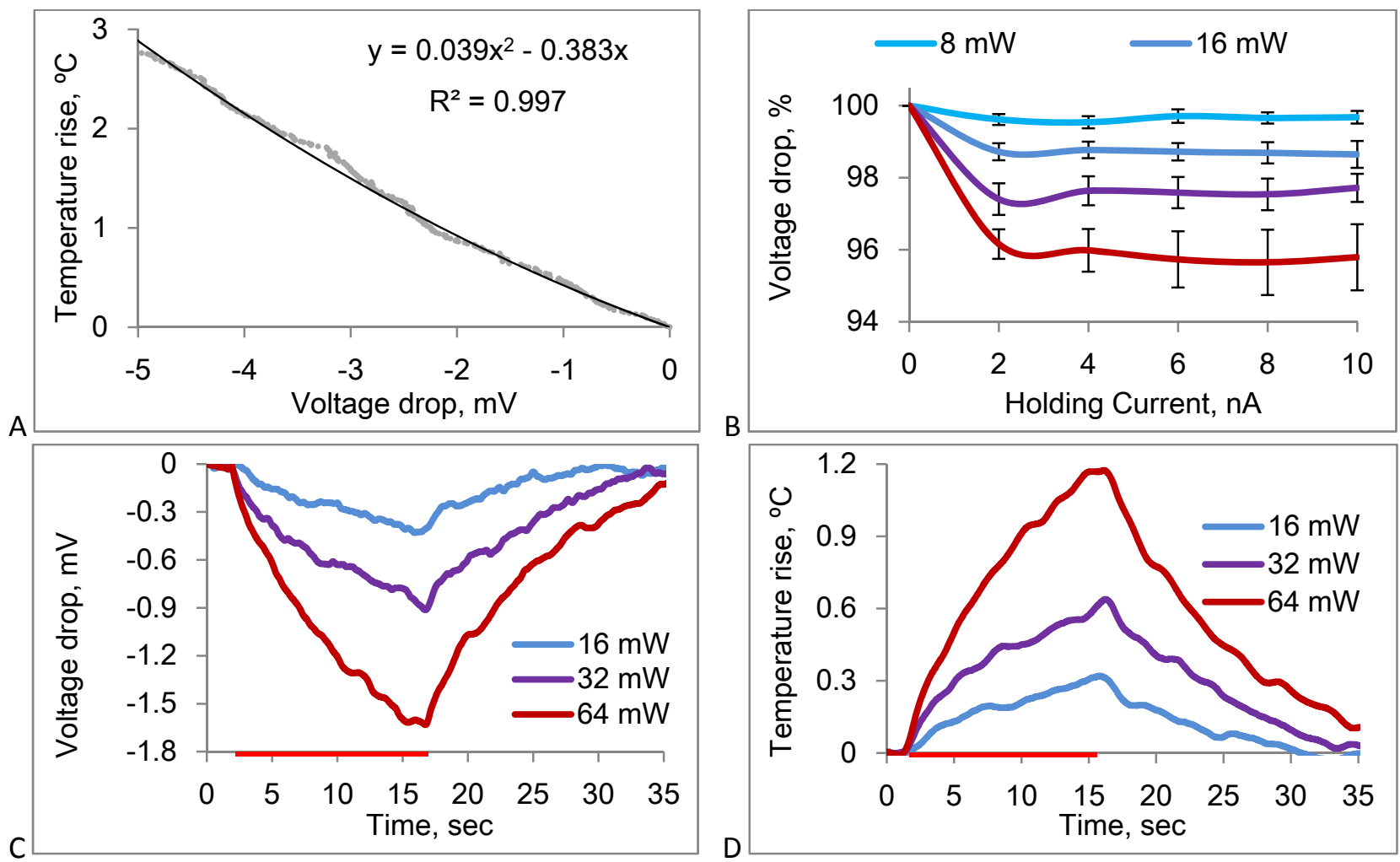

Figure 6. (A-B): Effects of general bath heating (A) and MMW irradiation (B) on the voltage drop inside the glass electrode relative to the baseline junction potential. Values refer to the power at the end of the waveguide port. In (B), the voltage drop is presented as the ratio of the value at different holding currents versus the value at the holding current of $0 \mathrm{nA}$. (C-D): Representative time-course of the voltage drop (C) and estimated temperature rise (D) during 15-sec-long MMW irradiation of the glass electrode $(R=26 \mathrm{M} \Omega)$. The horizontal red bar indicates the duration of MMW irradiation. 